\title{
Consumo de Álcool e Atenção Primária no Interior da Amazônia: sobre a Formação de Médicos e Enfermeiros para Assistência Integral
}

\author{
Alcohol Abuse and Primary Care in the \\ Amazon: Training Nurses and Physicians for \\ Comprehensive Care
}

Rodrigo Otávio Moretti-Pires Clarissa Mendonça Corradi-Webster ${ }^{I I}$ Erikson Felipe Furtado II

\section{PALAVRAS-CHAVE: \\ - Alcoolismo \\ - Atenção Primária à Saúde \\ - Formação de Recursos Humanos}

\section{KEYWORDS:}

- Alcoholism

- Primary Health Care

- Human Resources Formatio

Recebido em: 30/11/2010

Aprovado em: 18/01/2011

REVISTA BRASILEIRA DE EDUCAÇÃO MÉDICA
${ }^{I}$ Universidade Federal de Santa Catarina, Florianópolis, SC, Brasil.

"I Universidade de São Paulo, Ribeirão Preto, SP, Brasil.

\section{RESUMO}

O uso problemático de álcool (UPA) é um dos principais problemas de saúde pública na lista de prioridades da Estratégia de Saúde da Família (ESF). Este artigo objetiva investigar a visão de enfermeiros e médicos de Saúde da Família sobre o UPA, focando a compreensão que trazem sobre esta temática e o modo como é trabalhada no dia a dia dos serviços da região amazonica. Trata-se de um estudo qualitativo, com 14 trabalhadores da ESF, médicos e enfermeiros recém-formados de um município do interior do Amazonas. Foram utilizados dois grupos focais. Os dados foram analisados por meio de análise temática, a partir dos princípios do SUS e da formação de recursos humanos em saúde. Os profissionais identificaram os fatores de risco para o UPA, mostraram conhecer as consequências para o indivíduo e para a sociedade, e descreveram os critérios utilizados pelos sistemas de classificação de doenças, mas não sabiam como atuar sobre esta questão, indicando problemas na formação universitária.

\section{ABSTRACT}

Alcohol abuse is one of the main public health problems on the list of priorities in Brazil's Family Health Strategy. The objectives of this study were to learn the views of family health nurses and physicians concerning alcohol abuse, focusing on their understanding of this issue and the way it is handled routinely by health services in the Amazon. This was a qualitative study with 14 recently graduated nurses and physicians from the Family Health Strategy in a county in the interior of the State of Amazonas. Two focus groups were used. The data were analyzed through thematic analysis, based on the principles of the Unified National Health System (SUS) and human resources training. The health professionals identified risk factors for alcohol abuse, were aware of the consequences for the individual and society, and described the criteria used by disease classification systems, but they did not know how to act on this issue, thus revealing gaps in the respective university training. 


\section{INTRODUÇÃO}

Desde 1988, quando foi promulgada a nova Constituição, no sistema de saúde do Brasil, o direcionamento das ações de saúde visa à universalização do acesso a todos os cidadãos e a uma visão integral de saúde ${ }^{1}$. Na década de 1990, ocorreram mudanças significativas no modelo de atenção, preconizando a Saúde da Família como principal conformação da Atenção Primária e do acesso ao sistema de saúde pelos cidadãos. Este modelo se fundamenta numa visão ampliada do processo saúde-doença, em clara dicotomia com a perspectiva biomédica, principalmente por primar pela intersetorialidade das ações de saúde e pela atuação da equipe de saúde focada na família e não no indivíduo ${ }^{1,2}$. Outras características que deveriam estar presentes nesse modelo são: o planejamento das ações em conformidade e proximidade com a realidade da população para a qual se prestam serviços; a participação dos diversos atores sociais nesse processo; a capacitação permanente da equipe de saúde com base nas questões e problemas de saúde que emergem no dia a dia do trabalho junto à comunidade ${ }^{3}$.

Longe de ter constituído um processo de implementação fácil, esse novo modelo de atenção encontra inúmeras dificuldades para se tornar efetivo segundo o que se preconiza. A formação do médico e do enfermeiro ainda está pautada em um currículo biomédico, que enfoca a doença e não a saúde ${ }^{4}$. Trata-se de um modelo fragmentado e focado na formação em especialidades, cuja composição desconsidera temáticas transversais, como aspectos sociais dos usuários, política, sexo, violência, uso de drogas licitas e ilícitas. Estas questões, quando aparecem, são trabalhadas apenas por um conteúdo reducionista e por meio dos protocolos rígidos de diagnóstico e intervenção medicamentosa, que deixam escapar aspectos sociais, relacionais e contextuais, de entendimento necessário a uma efetiva e eficiente atuação do profissional de saúde no sentido da promoção de saúde. Este quadro está bem distante do preconizado para este modelo e do foco do sistema público de saúde nos novos paradigmas assumidos pelo SUS a partir de sua implementação. Ressalta-se: o problema apontado está muito ligado ao fato de que os recursos humanos inseridos no SUS foram formados em Instituições de Ensino Superior que pautam seus currículos de graduação em um paradigma antigo e divergente do talhe necessário à prática dos princípios do SUS, pautado na especialização e nas temáticas tradicionais de saúde, que baseiam suas ações na perspectiva biomédica e na doença propriamente dita.

Estas considerações se tornam ainda mais contundentes quando se examina de perto a proposta idealizada pelo Ministério da Saúde para a Estratégia de Saúde da Família: marcada por profunda e gradativa reorganização do processo de pro- dução dos serviços de saúde, a implementação das diretrizes da Saúde da Família se pauta na efetivação dos princípios do SUS $^{5-7}$, valorizando o enfoque do serviço de saúde rearticulado para implementar ações que recaiam sobre os determinantes de saúde e não apenas sobre a doença ${ }^{8,9}$. Isto incide sobre o papel do paciente, ressignificado e valorizado inclusive pela implementação do controle social, garantindo participação junto à gestão do sistema, em busca de intervenção nos problemas mais significativos de saúde pública para cada população ${ }^{10}$.

Neste tocante, o uso problemático de álcool vem se apresentando como um importante problema de saúde pública da atualidade ${ }^{11}$, pois se estima que se relacione à etiologia de 1,5\% das mortes, em termos mundiais ${ }^{12}$. Com relação ao Brasil, um estudo divulgado pelo Observatório Brasileiro de Informações sobre Drogas (Obid) ${ }^{13}$ aponta que $65 \%$ dos homens adultos e $41 \%$ das mulheres adultas bebem pelo menos uma vez ao ano, um total de $52 \%$ dos brasileiros acima de 18 anos. Entre os homens adultos, $11 \%$ bebem todos os dias e $28 \%$ consomem bebida alcoólica de uma a quatro vezes por semana. Quanto ao número de doses consumidas por ocasião, 38\% dos homens consumiram cinco ou mais doses, quantidade potencialmente prejudicial e que também indica alta tolerância à substância. Apesar da conscientização de que o uso de álcool ocasiona problemas pessoais, $8 \%$ relataram ter tido problemas no trabalho e mesmo assim continuaram fazendo uso da substância; $25 \%$ continuaram a beber, mesmo tendo problemas familiares consequentes deste consumo. Em termos gerais, 3\% do total da população com mais de 18 anos faz uso nocivo desta substância e $9 \%$ e é dependente de bebidas alcoólicas, segundo os dados do Obid $^{13}$.

Uma importante consideração é que o álcool, historicamente, adquiriu importância relacionada ao consumo oportunizado pelos aspectos culturais e simbólicos, na maioria das populações ${ }^{13}$. O padrão de uso revela muito destes, indo ao encontro de outra perspectiva da temática: a relação entre uso e danos agudos e crônicos pela toxicidade em órgãos e sistemas corporais, assim como o risco de provocar intoxicação aguda e dependência ${ }^{14}$.

Neste sentido, o enfoque na Atenção Primária é necessário. Segundo a OMS, o monitoramento do uso do álcool é relevante pela magnitude da abrangência dessa substância como instrumento de socialização. E não apenas a dependência estrita de álcool, mas também os quatro padrões de consumo que podem ser identificados pelos profissionais de saúde: uso de baixo risco (dentro dos limites considerados como de baixo risco); uso de risco (acima dos limites, mas sem presença de danos); uso nocivo (acima dos limites, com a presença de danos); e dependência (de acordo com os critérios da CID-10). 
Mais importante do que simplesmente estabelecer parâmetros, estes quatro padrões permitem intervenções direcionadas a cada um, oportunizando aportes teóricos e processuais para que os profissionais percebam os limites entre os padrões e ajam de acordo com eles ${ }^{15}$.

A formação dos profissionais de saúde para atuar frente ao uso problemático de álcool parece influenciada por diversos modelos que visam compreender esse uso em nossa sociedade. Alguns dos modelos mais utilizados são: ético-legal, moral, médico, psicológico, social e de múltiplos fatores de risco ${ }^{16}$. A atuação do profissional frente a esta problemática irá variar de acordo com o modelo adotado. Assim, se o profissional adotar o modelo moral, provavelmente não encarará o uso problemático de álcool como algo de sua alçada, mas, sim, da igreja ou de outros grupos que trabalhem com mudanças na índole individual.

Apesar dos princípios de saúde preconizados na Constituição vigente e do novo modelo de atenção que prima pela visão integral, particularmente pertinente à Estratégia de Saúde da Família, evidencia-se grande demanda por ações de saúde em regiões menos privilegiadas pelo acesso geográfico, como o interior da Amazônia brasileira. Esta demanda pode ser levantada por fatos históricos, como a implantação, em 2006, do Instituto de Saúde e Biotecnologia da Universidade Federal do Amazonas, com cursos de Nutrição, Enfermagem e Fisioterapia, no coração do Estado do Amazonas. Este instituto tem atividades no município de Coari, com 80 mil habitantes, dos quais $40 \%$ são moradores da área rural. Dista 390 quilômetros em linha reta de Manaus e 440 quilômetros no transcurso do rio, sendo o acesso geográfico exclusivamente pelas vias fluvial e aérea. O município tem uma rede de serviços de saúde com 11 Unidades de Atenção Primária, que funcionam oficialmente no modelo de atenção de Saúde da Família, e um hospital regional de média complexidade, com uma equipe de três psicólogos, mas nenhum psiquiatra.

Inexistem estudos sobre a formação dos médicos e enfermeiros que atuam em áreas com acesso geográfico prejudicado, como a região do interior da Amazônia, e sua adequação ao atendimento integral dos cidadãos que acessam o serviço público de saúde, em especial para temáticas que não têm espaço específico nos currículos de graduação, como a do uso problemático de álcool.

A proposta da Estratégia de Saúde da Família, preconizada pelo Ministério da Saúde, contém princípios como: (a) visão integral dos usuários; (b) planejamento local a partir das demandas da população adscrita à Unidade de Saúde da Família; (c) capacitação permanente dos profissionais para atuar nestas demandas específicas da população adscrita. Neste sentido, o presente trabalho teve por objetivo investigar a visão de enfermeiros e médicos de Saúde da Família sobre o uso problemático de álcool, focando a compreensão que trazem sobre esta temática e o modo como é trabalhada no dia a dia dos serviços públicos de saúde desta região amazônica.

\section{PERCURSO METODOLÓGICO}

Trata-se de um estudo qualitativo, com uso de entrevista de grupo focal e amostra selecionada entre os médicos e enfermeiros das equipes de Saúde da Família de Coari (AM), cuja característica relevante é terem se formado há menos de dois anos, refletindo sobremaneira o curso de graduação em que se formaram.

Participaram do estudo seis médicos (três mulheres e três homens) e oito enfermeiros (seis mulheres e dois homens), conforme recomendação da literatura para composição de grupos focais ${ }^{17,18}$. O número de participantes representou, respectivamente, $25 \%$ dos médicos e $30 \%$ dos enfermeiros da rede municipal de saúde. O critério de inclusão adotado foi: trabalhar em unidades de Atenção Primária que seguiam o modelo de Saúde da Família e concordar em participar da pesquisa. Os participantes foram recrutados individualmente, por meio da ida de um dos pesquisadores a cada um desses serviços. Todos os convidados aceitaram participar do estudo. Foram formados dois grupos focais, um para cada grupo profissional, com duração de uma hora cada, no período de novembro a dezembro de 2007. Estes grupos foram realizados em datas agendadas, em sala cedida pelo Instituto de Saúde e Biotecnologia da Universidade Federal do Amazonas, Coari (AM). A média de idade dos enfermeiros foi de 29 anos, e a dos médicos foi de 28 anos. Com relação ao tempo de formado, a média entre os enfermeiros foi de dois anos, e entre os médicos foi de seis meses. Quanto ao tempo de residência no município, todos os enfermeiros residiam havia mais de um ano, com média de um ano e meio, e todos os médicos estavam no município havia menos de seis meses.

No levantamento dos dados, foi utilizada a entrevista em grupo focal. Esta técnica já foi bem descrita na literatura ${ }^{18}$, sendo também empregada em outros contextos para avaliação da implementação de rotinas de rastreamento e intervenções breves sobre o uso problemático de álcool ${ }^{19-21}$.

Para esta entrevista, foi preparado um roteiro com os seguintes temas: aspectos da etiologia do uso problemático de álcool; a experiência de trabalhar com pacientes que fazem uso problemático de álcool; ações individuais e da equipe no manejo do uso problemático de álcool.

Os grupos focais tiveram, em média, uma hora de duração e foram gravados em áudio com uso de minigravadores. Os 
grupos foram conduzidos por um moderador e acompanhados por um observador que anotava suas impressões do grupo, assim como expressões dos participantes. Durante o grupo, ambos sumarizavam os apontamentos dos participantes. Ao término das sessões, os dois pesquisadores confeccionaram um relatório de consenso para auxílio na análise dos dados, conforme procedimentos estabelecidos pela literatura ${ }^{17,18}$. Tanto o moderador como o observador passaram por treinamento em condução de grupos focais e foram capacitados sobre a temática antes da realização dos encontros com os sujeitos.

O projeto de pesquisa foi aprovado pelo Comitê de Ética em Pesquisa com Seres Humanos da Universidade Federal do Amazonas (CAAE 0040.0.115.000-07). Todos os participantes registraram seu consentimento formal antes da participação nas entrevistas em grupo focal, assinando o Termo de Consentimento Livre e Esclarecido.

Todas as entrevistas foram transcritas na íntegra e lidas exaustivamente por dois pesquisadores que não se envolveram na coleta dos dados, de maneira independente e isolados um do outro, para que se familiarizassem com o conteúdo destas. Também realizaram individualmente um recorte das falas e a categorização do material, classificando as falas pertinentes à temática investigada, codificando por similaridade ou por divergência, estabelecendo categorias de análise. Do agrupamento das categorias semelhantes, estabeleceram-se Unidades de Análise, conforme orientação da literatura ${ }^{22-24}$. Após esta etapa, os dois pesquisadores, juntamente a um terceiro pesquisador do grupo de pesquisa, discutiram o produto das análises, obtendo o consenso registrado nos resultados. Este percurso foi similar ao descrito por outros autores para pesquisas que utilizam grupos focais como fonte de dados ${ }^{17,18}$. Os registros realizados pelo observador foram utilizados principalmente para clarificar o tom emocional das falas, importante na garantia da indexicalidade destas. Nos extratos selecionados para ilustrar a análise realizada, este tom emocional aparecerá entre colchetes ao final da fala, quando for necessário para auxiliar a compreensão desta.

\section{RESULTADOS}

\section{Perspectivas Gerais sobre a Temática}

Na exploração inicial do tema, perguntou-se aos profissionais o que pensavam a respeito do uso problemático de álcool. $\mathrm{O}$ grupo de enfermeiros enfatizou as causas que levariam a este consumo, dentre elas: valor social positivo dado ao consumo de bebidas alcoólicas; influência poderosa da mídia por meio das propagandas de bebidas alcoólicas (gerando valor social positivo); falta de apoio do poder público para criar políticas que fiscalizem e restrinjam este consumo.
Nas falas destes profissionais, percebeu-se a adoção de um discurso que abarca o modelo de fatores de risco, privilegiando os fatores sociais. Outro fator de risco abordado se refere aos fatores familiares, já que a família foi vista como tendo dificuldades na transmissão de valores morais positivos, servindo de modelo para perpetuar este uso problemático de álcool. A falta de uma religião também apareceu identificada como fator de risco, já que foi vista como influenciando o aumento do consumo, pensamento coerente com o modelo moral.

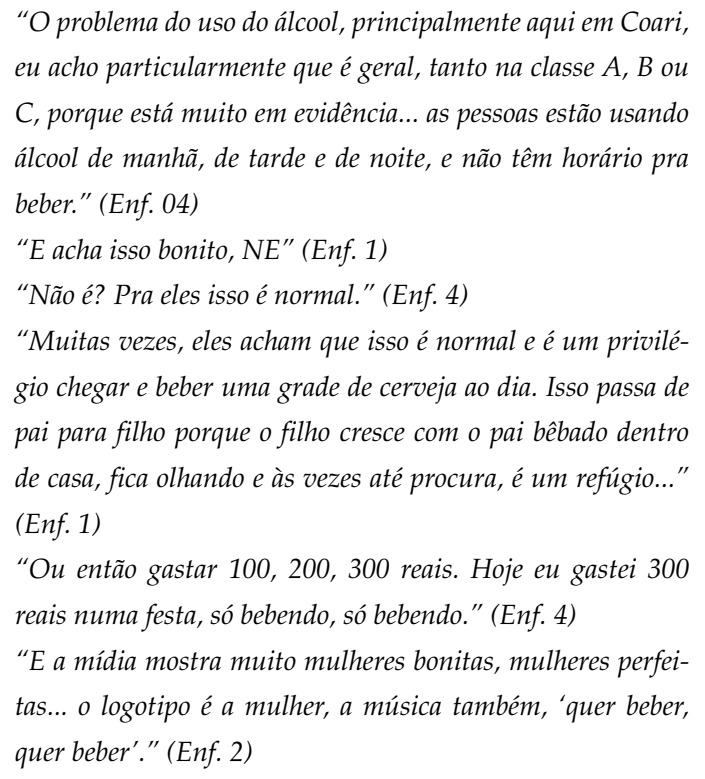

$\mathrm{Na}$ análise destas falas, percebe-se que a formação do enfermeiro na área de álcool e drogas vem sendo influenciada pela orientação de identificação de fatores de risco. Dentre estes, os sociais foram os mais ressaltados, em detrimento dos fatores de risco biológicos e psicológicos.

Já entre os médicos, nesta exploração inicial, prevaleceram falas que tratavam o uso problemático de álcool como uma doença que pode acarretar diversos prejuízos físicos e psicológicos, sendo um importante fator de risco para outras doenças. Nestas falas, percebe-se que a formação do médico propicia um olhar para o diagnóstico da doença e a reflexão sobre as implicações desta para a saúde física e psíquica do indivíduo.

\footnotetext{
"Eu acho que todos nós aqui consideramos o alcoolismo uma doença. Fala-se em vício, mas pode ser considerada uma doença que causa prejuízo não só físico como mental para todas as pessoas que estão envolvidas e convivem próximo à pessoa que sofre dessa doença." (Méd. 3)
} 


\section{Características do Uso Problemático de Álcool}

Quando apresentada aos enfermeiros, a temática "uso problemático de álcool" foi entendida como uma doença e classificada como "dependência do álcool". As características descritas para este padrão de uso foram: uso contínuo; sintomas de abstinência pela interrupção do consumo; dificuldades de controlar o consumo; ser reconhecido como problema pelas pessoas ao redor do usuário. Entretanto, este usuário foi descrito como de difícil adesão ao tratamento por não reconhecer que tem um problema e por ser constantemente influenciado pelos fatores de risco sociais, como a mídia.

"Porque, quando chega nessa fase, ele já não é ninguém mesmo, já não tem limites, ele mesmo já não se domina mais, quem domina na verdade é o corpo dele, ele tenta por necessidade; então, quando chega nessa fase, ele faz qualquer coisa, qualquer coisa mesmo." (Enf. 5)

Ao considerarem o uso problemático de álcool apenas como dependência, os enfermeiros descreveram perceber os pacientes como pessoas resistentes em aceitar o problema e com dificuldades para aderir ao tratamento. Com isto, se sentiam enfraquecidos e desmotivados para se envolver numa relação de ajuda com esta população.

"Que o pessoal que fala que ele mesmo está no limite dele, que ele se domina, mas na verdade todo mundo sabe que é uma doença, que na verdade ele precisa mesmo é de ajuda; então, nessa hora a família tem que entrar, porque se não, se a família dele não fizer nada, quem vai fazer?" (Enf. 5)

Para os médicos, o uso problemático de álcool foi descrito como aquele que deixou de ser um uso social, sendo caracterizado como um tipo de consumo que traz prejuízos ao indivíduo e que envolve a presença de sintomas de abstinência:

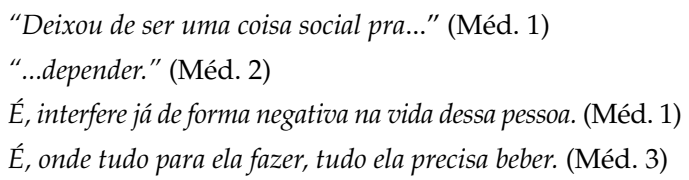

Nas falas dos médicos, como foi dada ênfase aos prejuízos causados pelo consumo de álcool, parece haver uma abertura para a percepção do usuário nocivo. Ao falarem sobre os danos causados pelo consumo de álcool, parecem aproximar este consumo do trabalho que realizam como profissionais de saúde. Entretanto, esta ênfase dada às consequências é explicada ao dizerem que o contato que tinham com pessoas que faziam uso problemático de álcool se dava apenas nos plan- tões em unidades de emergência, quando recebiam pacientes que sofreram acidentes automobilísticos ou se envolveram em brigas devido ao consumo excessivo de álcool. Percebe-se que a visão do uso problemático como fator de risco para outros problemas não os colocava no papel de agentes de prevenção, ou seja, não influenciava para que adotassem posturas e práticas preventivas. É o que ilustra o extrato a seguir.

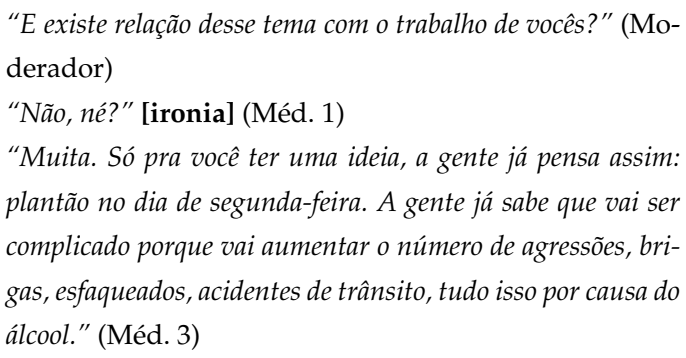

\section{Identificação na Atenção Primária}

Ambas as categorias profissionais concordaram em que não identificavam o uso problemático de álcool. Os enfermeiros explicaram que não recebiam procura espontânea e em momento algum da entrevista, mesmo quando perguntados sobre como identificavam este consumo, falaram sobre a identificação. Mantiveram durante toda a entrevista a postura de espera pela demanda do paciente.

Os médicos relataram que apenas identificavam casos em que os pacientes estavam intoxicados, mas reconheciam que seria importante que a Atenção Primária estivesse comprometida com a busca ativa. Entretanto, colocaram a responsabilidade por esta atividade em outros profissionais, culpabilizando-os por não fazerem e por não terem formação para isto, como é possível observar no extrato a seguir.

"Talvez seja até uma deficiência do PSF esse fato de não se levar realmente o alcoolismo como uma doença, tanto é que a gente sabe que é comum, mas não existe essa busca ativa. É por parte de toda a equipe, não só de agentes de saúde, médicos. Mas não existe, não se vai numa casa, e o agente de saúde fala: nós temos uma pessoa aí que sofre de alcoolismo." (Méd. 1)

"É!!" (Méd. 3)

"Então vamos pedir pro médico, pro enfermeiro fazer uma visita. Isso não acontece. Isso é até uma deficiência. Que eu acho." (Méd. 1)

"Mas esse problema..." (Méd. 4)

"A ignorância dessas pessoas também, que muitas vezes nem veem isso como um problema."(Méd. 2)

"Exatamente." (Méd. 4)

"Eles acham isso normal..." (Méd. 2) 


\section{Intervenção}

Quanto à intervenção nestes casos, os enfermeiros pareciam se sentir "desempoderados" para agir de forma ativa. Diante de todos os fatores de risco sociais considerados por eles e do papel relevante que dão à mídia na sedução para o consumo, se sentiam sós no trabalho de orientação para reduzir ou cessar o consumo.

"Olha, eu me sinto uma formiga por tamanha problemática." (Enf. 4)

Sugerem que a educação em saúde faz parte de suas atividades, mas em seguida propõem a realização de intervenções preventivas em escolas e consideram que os profissionais de saúde deveriam dar exemplo com seus próprios hábitos de vida, conforme ilustrado no extrato a seguir.

"Como vocês intervêm sobre essa problemática?" (Moderador) "Eu vejo assim como elas falaram, se não tiver o apoio dos governantes, futuramente vai ser uma população de alcoólatras, uma população muito grande de pessoas viciadas, dependentes do álcool, do cigarro, da droga, principalmente da droga, porque a droga está crescendo muito em Coari, muito." (Enf. 3) "Coari é vista lá fora como a cidade da prostituição e da droga." (Enf. 1)

"O que eu tive no serviço de saúde pública e parte da nossa atividade é principalmente educação em saúde." (Enf. 3)

"As escolas seriam os pontos primordiais, porque é onde está o adolescente, é onde está a garota, o garoto ou até mesmo o professor que está convivendo com o aluno, que fuma, que bebe. Triste é ver um profissional da saúde, médico, dentista, fumando." (Enf. 2)

"Eu acho muito feio, porque nós, que fizemos o juramento de salvar vidas e de tratar as pessoas, deveríamos dar bom exemplo como profissionais da saúde. Isso é muito feio, isso é um dos princípios que marcam os profissionais de saúde, que deveriam dar bons exemplos e não dão com a criança, até com o adolescente mesmo, de ficar fumando na frente dos adolescentes, bebendo; deviam ter cuidado com isso, eu acho que tem que ter, eu acho que é uma coisa feia que pesa pra caramba." (Enf. 2)

Os médicos reconheceram que não faziam intervenção, a não ser nos casos que recebiam na emergência, onde o foco era a resolução do problema causado pelo consumo da bebida. Como resolução para esta falta de envolvimento com o tema, os entrevistados sugeriram que o governo elaborasse políticas de prevenção.

"Eu acho que a nossa intervenção no problema do alcoolismo é pouca. Nossa intervenção é mínima porque a gente não consegue..." (Méd. 1)
"A gente não entra na causa, a gente entra na conseqüiência, entendeu?" (Méd. 2)

Ambas as categorias profissionais concordaram em que este tema está relacionado ao trabalho que realizam, mas encontraram dificuldades de explicar qual seria esta relação. Disseram que não têm experiências de pacientes que procuram ajuda para esta problemática, e os médicos relataram que o contato com esta temática se dá no serviço de emergência, pontuando como é difícil para eles lidar com o grande número de pacientes que chega por problemas consequentes ao uso problemático de álcool nos plantões em dias de comemorações.

\section{DISCUSSÃO}

O discurso dos médicos, ao considerarem o uso problemático de álcool como doença e como fator de risco para outras doenças, parece aproximar este tema de seu campo de atuação, uma vez que, como médicos, devem identificar fatores de risco para doenças e tratar outras já presentes. No entanto, esse discurso tem sempre um caráter biomédico, centrado na doença e na cura, distanciando da perspectiva da atuação intersetorial e da promoção e prevenção de saúde.

A desarticulação entre o novo paradigma dos princípios preconizados para o trabalho na ESF e a formação pelo paradigma biomédico do profissional que atua neste modelo é uma questão amplamente apresentada pela literatura em diversos campos ${ }^{4}$. No que concerne à temática do álcool, poucos estudos abordam este nó crítico, que tem sua importância investigada por diversos autores. É apresentado como um dos principais problemas de Saúde Pública no País, por constituir um fator determinante de mais de $10 \%$ de toda a morbidade e mortalidade do Brasil na atualidade. Em termos mundiais, em 2000 , respondeu por $4 \%$ de toda a morbidade e mortalidade, com tendência a ascensão. Neste sentido, intervir na temática do álcool é importante, já que pode direcionar programas de prevenção que visem reduzir problemas decorrentes do uso ${ }^{25}$.

Existem propostas para utilizar instrumentos de rastreamento deste problema na Atenção Primária. O Questionário de Identificação de Transtornos Causados pelo Consumo de Álcool (Audit) e o Cut Down/Annoyed/Guilty/Eye-Opener Questionnaire (Cage) são exemplos de instrumentos para este fim, sendo de fácil aplicação e utilização ${ }^{26}$. Portanto, há estratégias que podem ser empregadas.

Também a importância junto à Atenção Primária tem sido destacada nos debates acerca dos serviços de saúde mental em todo o mundo, pautando-se na premissa de que se podem resolver diversos problemas em saúde mental nesse nível de assistência, principalmente no que se refere ao desenvolvimento 
de ações preventivas e à promoção da saúde mental ${ }^{27}$. Neste sentido, há confluência entre a proposta da ESF e a discussão da temática do álcool em seu processo de produção.

No âmbito da ESF, as ações nesta temática deveriam ir além das curativas e das focadas em terapias medicamentosas, considerando-se o complexo usuário-família como agente fundamental do cuidado, valorizando os aspectos intersubjetivos, sociais e políticos do entorno socioeconômico e cultural dos usuários ${ }^{28}$. Para isto, é necessário um trabalho integrado entre os profissionais de ESF, tanto quanto o trabalho integrativo dos aportes de conhecimento destes e a construção de um objeto de trabalho integrador, em que os aspectos envolvidos na temática do uso problemático de álcool podem contribuir significativamente neste construir.

No entanto, de forma dicotômica com estes direcionamentos, o que se encontrou no universo empírico investigado mostra que, de maneira geral, há certa fragilidade no discurso dos profissionais no que se refere à operacionalização de intervenções voltadas ao consumo de álcool. Os enfermeiros utilizam o discurso dos fatores de risco sociais, mas este não parece ser útil no processo de empoderamento destes profissionais para a ação, já que se sentem pequenos frente à mídia e à mudança de valores da sociedade e da família. Não sabendo como intervir como profissionais, buscam a intervenção como "indivíduos que dão bons exemplos". Isto chama a atenção para a formação oferecida aos profissionais de saúde, pautada, muitas vezes, na discussão de fatores de risco ou na busca das causas, sem formar o profissional para agir sobre elas. Isto também apareceu no grupo dos médicos, que, embora considerassem o uso problemático de álcool como fator de risco para outras doenças, disseram que não faziam busca ativa, não se sentiam preparados para atuar preventivamente e ficavam frustrados por agir apenas sobre as consequências do consumo.

Ao mesmo tempo, ambos os grupos de profissionais sentem dificuldade de trabalhar sem uma rede, sem equipe multiprofissional, sem apoio governamental e sem equipamentos de saúde e sociais. Eles não identificam e não intervêm diretamente sobre o assunto e parecem não saber como fazer, não saber ao certo seu papel, esperando que haja uma política para isto, que a mídia mude e/ou que o usuário procure o profissional motivado para mudar.

É importante destacar também que a literatura vem chamando a atenção para dificuldades no trabalho em saúde em algumas regiões do Brasil, como no interior do Amazonas. Parte destas dificuldades se daria pela pouca experiência dos profissionais e pelo pequeno vínculo destes com o território, já que muitos vão trabalhar no interior do Estado logo após concluir a graduação enquanto aguardam outros empregos na capital ou em outras localidades ${ }^{29}$. Com isto, desconhecem o território onde trabalham e têm poucas chances de construir vínculos com a população e com outros serviços, o que pode acarretar prejuízos à assistência.

Tomando as ações próprias da ESF como ponto de partida, mesmo nos casos em que os profissionais não estão preparados para ações de intervenção na temática do álcool, por tentarem romper com o modelo biomédico através da consideração do contexto social do paciente como importante para a prática de saúde, em que todo problema presente - como o do uso problemático de álcool - deveria ter encaminhamento a outros níveis de atenção, a ESF deveria se articular com a rede de saúde mental ${ }^{28-33}$. Mas não é o que foi encontrado na presente pesquisa. Na realidade, nem mesmo os profissionais debatem o que fazer quando a temática emerge, ferindo de forma contundente o princípio da integralidade na assistência. Indício da fragmentação do cuidado e do pautar da ESF no modelo biomédico, na medida em que a equipe enfoca doenças físicas dos usuários, sem atuar nas questões de saúde mental ${ }^{28}$.

Outra perspectiva teórica importante se refere ao foco na temática do uso de álcool como doença, que reivindica, de certa forma, a legitimidade do poder social dos profissionais de saúde, como se a temática se referisse tão somente ao adoecer e ao curar $^{33}$, e não a um laço mais profundo, relacionado aos aspectos do álcool ligados à socialização, como, por exemplo, a diferença entre o bebedor de bar e o bebedor solitário, manifestações distintas do fenômeno ${ }^{34}$; ou mesmo a questão do uso de álcool como emblema de status, controle de comportamento e minúcias do poder ${ }^{33}$.

As intervenções breves podem complementar as atividades assistenciais habituais e podem ser inseridas na rotina usual de atendimento, ocupando um tempo mínimo, utilizando recursos didáticos para obter rapidamente maior nível de informação sobre os problemas atuais do paciente, avaliando e fazendo emergir a motivação para mudança, preparando-o para tomar decisões. Assim, por exemplo, um médico da Estratégia de Saúde da Família poderá incorporar à consulta de rotina a triagem para identificar pacientes com problemas relacionados ao uso de álcool, incluindo os casos de risco em seu esquema usual de seguimento e acompanhamento ${ }^{35}$.

A importância do alcoolismo para a Atenção Primária é significativa, uma vez que o tema consta entre os dez mais importantes problemas de saúde da lista de prioridades do Programa Nacional de Saúde da Família. A equipe de Saúde da Família é apresentada como central nas discussões sobre a capacitação para a intervenção nesta temática, mediante estratégias de rastreamento e intervenções breves para o uso problemático 
de álcool na rotina do atendimento oferecido na $\mathrm{ESF}^{35}$, haja vista a importância da temática e o fato de que as intervenções breves objetivam reduzir o risco de dados do uso de álcool ${ }^{35-37}$.

Em outra perspectiva, cabe debater também certo silêncio dos depoentes em relação ao trabalho em equipe, que deveria se pautar em projeto assistencial comum, em que os diversos profissionais de Saúde da Família desenvolveriam ação de interação entre si e a comunidade ${ }^{38}$. Temas transversais, como o enfocado aqui, deveriam permear constantemente o processo de trabalho destes profissionais, inserindo-os na perspectiva coletiva do trabalho em ESF, o que não foi apresentado como característica do trabalho em Saúde da Família no interior da Amazônia, segundo os entrevistados.

Na medida em que a formação e o perfil profissional são requisitos fundamentais para uma nova perspectiva de atenção no SUS como um todo e na Saúde da Família em particular, os resultados debatidos no presente artigo apontam a premente necessidade de adotar caminhos para a implementação efetiva de políticas e mecanismos que viabilizem a educação permanente. Sendo um dos instrumentos que valorizam a mudança na Atenção Primária no País, cada equipe deveria ser capacitada em saberes que não foram trabalhados durante a graduação ou que não garantiram formação satisfatória para o que a prática cotidiana demanda ${ }^{4,38-41}$. Dado o vulto da temática do álcool no dia a dia destes profissionais, evidencia-se a necessidade de estratégias que viabilizem a capacitação nesta e em outras temáticas transversais. Particularmente na temática do UPA, existem estratégias consagradas e ajustadas à realidade da Atenção Primária, com baixo custo e fácil implementação ${ }^{15,21,26,35-37}$.

A questão da decisão política das gestões municipais, estaduais e federal se mostra, portanto, fundamental para estabelecer essas estratégias de intervenção, de forma a superar a situação identificada no presente artigo, relacionada à formação destes profissionais.

Não apenas em termos da região amazônica, mas no âmbito da Atenção Primária brasileira, há que se lembrar que é papel do SUS a proposição de processos que se relacionem com a educação permanente das equipes de saúde.

\section{CONSIDERAÇÕES FINAIS}

O despreparo dos profissionais de nível superior para a assistência integral dos usuários está imerso no contexto apresentado no presente trabalho, apesar da importância da saúde mental e da temática do uso de álcool nesta assistência.

A formação dos profissionais de saúde ainda carece de foco a respeito da atuação prática destes após sua graduação. E mais: o ensino universitário tem o papel de formar para o SUS, conforme a legislação que o regulamenta no País.
Neste estudo, os profissionais identificaram os fatores de risco para o uso problemático de álcool, conheciam as consequências desse consumo para o indivíduo e para a sociedade, descreviam os critérios utilizados pelos sistemas de classificação de doenças (CID-10, DSM-IV), mas não sabiam como atuar sobre esta questão.

São necessárias mudanças na estrutura de formação e capacitação dos futuros médicos e enfermeiros, para uma atuação genuína e condizente com os princípios do SUS e a prática em ESF. A inserção de estratégias de tratamento breve na formação de profissionais parece importante para subsidiar as ações, principalmente em saúde mental, em que grande parte da complexidade do atendimento reside na comunicação. Este trabalho identificou certo despreparo na atuação de médicos e enfermeiros de Saúde da Família do interior da Amazônia frente à identificação e intervenção em questões relativas ao uso de álcool na população adscrita.

A capacitação permanente das equipes com base na realidade local e nos problemas da população assistida deveria ocorrer mesmo no interior da Amazônia, tendo em vista a regulamentação da Saúde da Família no Brasil.

\section{REFERÊNCIAS}

1. Carvalho AI, Westphal M, Lima VLGP. Historical overview of health promotion and health promotion evaluation in Brazil. Prom Health Educ. 2007;(Suppl 1): 7-12.

2. Atkinson S, Cohn A, Ducci ME, Gideon J. Implementation of promotion and prevention activities in decentralized health systems: comparative case studies from Chile and Brazil. Health Prom Internat. 2005;20(2):167-75.

3. Elias PEM, Cohn A. Health reform in Brazil: lessons to consider. Am J Public Health. 2003;93 (1):44-8.

4. Moretti-Pires RO. O pensamento crítico social de Paulo Freire sobre humanização e o contexto da formação do enfermeiro, do médico e do odontólogo. Ribeirão Preto; 2008. Doutorado [Tese] - Escola de Enfermagem de Ribeirão Preto da Universidade de São Paulo.

5. Campos CEA. O desafio da integralidade segundo as perspectivas da vigilância da saúde e da saúde da família. Ciênc Saúde Colet. 2003;8(2):569-84.

6. Gerschman S. A democracia inconclusa: um estudo da reforma sanitária brasileira. Rio de Janeiro: Ed. Fiocruz; 2004.

7. Vasconcelos CM. Paradoxos da mudança no SUS Campinas; 2005. Doutorado [Tese] - Universidade Universidade Estadual de Campinas.

8. Campos GWS. O SUS entre a tradição dos Sistemas Nacionais e o modo liberal-privado para organizar o cuidado à saúde Ciênc Saúde Colet. 2007;12:1865-74. 
9. Campos CMS, Bataiero MO. Health needs: an analysis of Brazilian scientific literature from 1990 to 2004. Interface Comun Saúde Educ. 2007;11(23):605-18.

10. Aciole GG. On pedagogical dimensions to citizenship building on social control practice. Interface Comun Saúde Educ. 2007;11(23):409-26.

11. Fligie NB, Pillon SC, Dunn J, Laranjeiro R. The frequency of smoking and problem drinking among general hospital impatients in Brazil: using the AUDIT and Fagerström questionnaires. São Paulo Medical Journal. 2000;11(5):139-43.

12. World Health Organization. The World Health Report 2001 - Mental Health: New Understanding, New Hope [online]. [acesso em 26 set. 2006]. Disponível em:<http:/ / www.who.int./whr2001.

13. Laranjeira R, Pinsky I, Zaleski M, Caetano R. I Levantamento Nacional sobre os padrões de consumo de álcool na população brasileira. Brasília: Secretaria Nacional Antidrogas; 2007.

14. Laranjeira R, Romano M. Consenso brasileiro sobre políticas públicas do alcool. Rev Bras Psiquiatr. 2004;26(Supl.1):68-77.

15. Babor TF, Higgins-Biddle JC. Brief intervention for hazardous and harmful drinking: a manual for use in primary care. Geneva: WHO; 2003

16. Pillon SC, Luis MAV. Modelos explicativos para o uso de álcool e drogas e a prática da enfermagem. Rev Lat Am Enfermagem. 2004;12(4):676-82.

17. Morgan D. Focus Group as qualitative research. 2nd ed. Sage Publications; 1997.

18. Krueger RA. Focus Groups. A practical guide for applied research. Thousand Oaks: Sage Publications; 1994.

19. Aalto M, Pekuri P, Seppa K. Obstacles to carrying out brief intervention for heavy drinkers in primary health care: a focus group study. Drug Alcohol Rev. 2003;22:169-73.

20. Rush BR, Powell LY, Crowe TG, Ellis K. Early interventions for alcohol use: family physicians' motivations and perceived barriers. CMAJ. 1995;152:863-69.

21. Corradi-Webster CM, Minto EC, Aquino FMC, Abade F, Yosetake LL, Gorayeb R, et al. Capacitação de profissionais do Programa de Saúde da Família em estratégias de diagnóstico e internvenções breves para o uso problemático de álcool. Rev Eletrônica Saúde Mental Álcool e Drogas. 2005;1(1):1-10.

22. Minayo MCS. O desafio do conhecimento: pesquisa qualitativa em saúde. 6a Ed. São Paulo:Hucitec; 1999.

23. Haguette TMF. Metodologias qualitativas na sociologia. 8a ed. Petrópolis: Vozes; 2001.
24. Leopardi M.T. Metodologia da pesquisa na saúde. 2 ed. Florianópolis: UFSC/Pós-graduação em Enfermagem; 2002.

25. Meloni JN, Laranjeira R. Custo social e de saúde do consumo de alcool. Rev Bras Psiquiatr. 2004;26(Supl.1):7-10.

26. Segatto ML, Pinsky I, Laranjeira R, Rezende FF, Vilela TR. Triagem e intervenção breve em pacientes alcoolizados atendidos na emergência: perspectivas e desafios. Cad Saúde Pública. 2007;23(8):1753-62

27. World Health Organization. Organization of mental health services in developing countries. Sixteen report of WHO Expert Committee on Mental Health. Geneva: WHO; 1975.

28. Nunes M, Jucá VJ, Valentim CPB. Ações de saúde mental no Programa Saúde da Família: confluências e dissonâncias das práticas com os princípios das reformas psiquiátrica e sanitária. Cad Saúde Pública. 2007;23(10):2375-84.

29. Faria IBR, Ventura JC, Silva DE, Moretti-Pires RO. O processo de trabalho em saúde da família no contexto do interior da Amazônia. Cogitare Enferm. 2010;15(2):231-72.

30. Brasil. Ministério da Saúde. Saúde da família: uma estratégia para reorientação do modelo assistencial. $2^{2}$ ed. Brasília: MS; 1998.

31. Tenório F. A psicanálise e a clínica da reforma psiquiátrica. Rio de Janeiro: Rios Ambiciosos; 2001.

32. Paim JS. Saúde da família: espaço de reflexão e contra-hegemonia. Interface Comun Saúde Educ. 2001;5:143-6.

33. Neves DP. Alcoolismo: acusação ou diagnóstico? Cad Saúde Pública. 2004;20(1):7-14.

34. Garcia AM. Os bares no contexto de um bairro popular [Relatório de pesquisa: $\mathrm{O}$ alcoolismo e a exclusão social]. Niterói: Universidade Federal Fluminense; 2000.

35. Marques ACPR, Furtado EF.. Intervenções breves para problemas relacionados ao álcool. Rev Bras Psiquiatr. 2004;26(supl.1):28-32

36. Babor TF, Longabaugh R, Zweben A, Fuller RK, Stout RL, Anton RF, et al. Issues in the definition and measurement of drinking outcomes in alcoholism treatment research. J Stud Alcohol Suppl. 1994;12:101-11.

37. Saunders JB, Aasland OG, Amundsen A, Grant M. Alcohol consumption and related problems among primary health care patients: WHO collaborative project on early detection of persons with harmful alcohol consumption - I. Addiction. 1993;88(3):349-62.

38. Araújo MBS, Rocha PM. Trabalho em equipe: um desafio para a consolidação da estratégia de saúde da família. Ciênc Saúde Colet. 2007;12(2):455-64.

39. Ceccim RB. Educação permanente em saúde: desafio ambicioso e necessário. Interface Comun Saúde Educ. 2005;9(16):161-8. 
40. Ceccim RB, Ferla AA. Residência integrada em saúde: uma resposta da formação e desenvolvimento profissional para a montagem do projeto de integralidade da atenção à saúde. In: Pinheiro R, Mattos R, org. Construção da integralidade: cotidiano, saberes e práticas em saúde. Rio de Janeiro: Abrasco; 2003. p.211-26.

41. Ceccim RB, Feuerwerker L. O quadrilátero da formação para a área da saúde: ensino, gestão, atenção e controle social. Physis Rev Saúde Colet. 2004;14(1):41-65.

\section{CONTRIBUIÇÃO DOS AUTORES}

Todos os autores contribuíram em todas as etapas, desde o projeto até a análise e redação final do artigo.
CONFLITO DE INTERESSES

Declarou não haver.

\section{ENDEREÇO PARA CORRESPONDÊNCIA}

Rodrigo Otávio Moretti-Pires

Campus Universitário

Trindade - Florianópolis

CEP 88040-970 — SC

E-mail: rodrigo.moretti@pq.cnpq.br 